\title{
Les proches
}

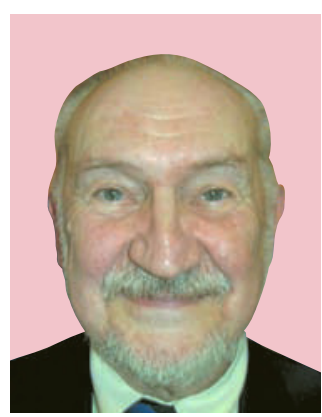

Jean-Pierre Escande

Administrateur national

de la Ligue contre le cancer

escandej@ligue-cancer.net

Abandonnons le discours convenu sur les proches. Qui sont-ils ? Leur place ? Leur rôle ? Que faire d'eux?

L'évolution du monde de la santé place le malade au centre du dispositif de soin. Cette pétition de principe est certes rassurante mais pas forcément efficace... Mais le proche, lui, n'est pas au centre du dispositif et ne souhaite pas l'être, ce n'est pas son rôle... alors que peut-il faire ou que peut-on faire de lui...?

\section{LE PROCHE, AUSSI DIFFÉRENT QUE LES MALADES}

La littérature en ce domaine est abondante, pour essayer de définir qui il est... un accompagnant ? Un soignant ? Un aidant ? Certains essaient même de définir le « proche idéal »... Idéal par rapport à qui ? Le proche est aussi différent que les malades, de nombreux critères peuvent être pris en considération, sexe, âge, lien parental, tout cela fait la diversité du proche. Mais la vraie question : s'est-on préoccupé un peu de lui, après avoir cherché à le définir ?

Il est ballotté au gré des interlocuteurs. On peut considérer à juste titre que la première mission d'un médecin c'est de suivre son malade, mais qui, à quel moment et comment se préoccupe-t-on des états d'âme du proche, de sa fatigue de ses difficultés avec les soignants ? Tantôt négligé, tantôt solli- cité pour un problème difficile, comment l'aide t-on à gérer son rapport avec le malade de plus en plus exigeant au fur et à mesure de l'aggravation de la maladie. Son isolement par rapport à l'entourage. Souvent sans droit de parole, assistant silencieux, sa présence est utile pour faire passer les messages douloureux, dont le médecin se débarrasse pour évacuer sa propre angoisse, mais je caricature.

Pour reprendre une attitude plus objective, je voudrais dire, sans occulter la souffrance du malade, que le proche lui aussi souffre de la maladie de celle ou celui qu'il ou qu'elle accompagne, des secrets qu'il conserve. De l'attitude qu'il doit avoir, pour ne pas alerter, inquiéter. Des difficultés d'accompagnement dans les soins à domicile, ce n'est certes pas un soignant mais c'est un observateur attentif de l'évolution de la maladie des nouvelles difficultés qui apparaissent. Ses problèmes d'accompagnement par manque de professionnalisme.

Il faut aussi compter avec la transformation de l'humeur ou du caractère de gravité de la maladie, comment faire face, à la fatigue accumulée liée à l'angoisse à la perturbation d'une vie, c'est tout cela la difficulté d'un proche.

Mais je n'apprends rien de nouveau, sauf peut-être demander de la part des soignants un peu d'empathie, un mot, une phrase d'encouragement, un mot, une phrase, donnant, sinon de l'espoir, un peu de sérénité. Alors, pour vous professionnels de santé, bien sûr le malade c'est votre "vie », mais n'oubliez pas trop ce proche qui est aussi près de vous.

\section{LES ATTITUDES DU PROCHE}

Sans vouloir m'appesantir sur la définition du proche qui, comme je l'ai dit précédemment, est aussi divers qu'il y a de malades, je voudrais revenir sur son attitude vis-àvis du malade et du soignant en général. Là encore, cette attitude est diverse, il recherche de l'information ou la rejette, comment le soignant peut-il comprendre ce proche qui justement ne lui est pas proche?
Le médecin en général connaît bien son malade et sa maladie, on peut le supposer, mais comment connaît-il le proche... C'est difficile, les attitudes étant là encore très variables... Celui qui recherche de l'information ou celui qui la fuit, celui qui s'enferme dans le silence par ce qu'envahit par l'angoisse, tout cela est complexe. Quel moyen de résolution de ce problème ? Je pense qu'il n'y a qu'une voie possible, c'est l'écoute, le regard pour l'autre, malheureusement la condition ultime de la résolution de ce problème c'est le TEMPS. Un BIEN précieux. Or, dans ce monde actuel de la santé, c'est ce qui manque le plus à tous, le temps qu'un médecin peut consacrer en plus de son malade, le temps que l'infirmier peut consacrer, le temps que le pharmacien peut consacrer au proche. Je crois que toutes les bonnes volontés de chacun, la formation des soignants, tout cela est anéanti si l'on ne dispose pas de suffisamment de temps car ce manque de temps, c'est l'excuse pour tout... manque d'information, manque d'écoute, manque empathie, manque d'explication, alors pour tous demandons du temps.

J'aborderai un dernier point c'est la fatigue du proche. Matthieu Ricard, dans un exposé sur l'homme dans les crises actuelles, parle de la "fatigue de la compassion » et du « burn out » du proche. Alors très égoïstement, je demande pour le proche le moyen d'avoir un répit, un espace de " revitalisation " d'aide, de reprise en main et, là encore, un peu de temps.

Pour finir, je reviendrai sur le sujet récurant de la formation des soignants à l'écoute. Cette question pour moi fait débat entre la fameuse thèse de l'inné et de l'acquis ; plus que de la formation donnons-nous du temps de réflexion sur le métier de soignant et je crois que cela serait supérieur à toute formation trop contraignante. Le proche est certes près de son «malade » mais il est aussi près des soignants et, comme le malade, il demande votre aide. Un peu de compassion, un peu d'empathie, un peu d'écoute et bien sûr un peu de temps... Est-ce un rêve ?• 